\title{
Genome-wide association scans for idiopathic osteonecrosis of the femoral head in a Korean population
}

\author{
SEUNG-HOON BAEK ${ }^{1}$, KANG-IL KIM ${ }^{2}$, KYUNG-SIK YOON ${ }^{3}$, TAE-HO KIM ${ }^{4,5}$ and SHIN-YOON KIM ${ }^{1,5}$ \\ ${ }^{1}$ Department of Orthopedic Surgery, Graduate School of Medicine, Kyungpook National University, \\ Daegu 41944; Departments of ${ }^{2}$ Orthopedic Surgery and ${ }^{3}$ Biochemistry and Molecular Biology, School of Medicine, \\ Kyung Hee University, Seoul 02447; ${ }^{4}$ Biomedical Research Institute, Kyungpook National University Hospital; \\ ${ }^{5}$ Skeletal Diseases Genome Research Center, Kyungpook National University, Daegu 41940, Republic of Korea
}

Received November 13, 2015; Accepted November 11, 2016

DOI: $10.3892 / \mathrm{mmr} .2016 .6036$

\begin{abstract}
Osteonecrosis of the femoral head (ONFH) is a multifactorial disease and is associated with genetic predisposition, and exposure to certain risk factors. In particular, idiopathic ONFH in twins and the clustering of cases in families have indicated that genetic factors are involved. However, the majority of cases of ONFH are sporadic and various studies have demonstrated that differences in the study design and/or the ethnic groups analyzed leads to different results. The present study performed one of the first genome-wide association studies to identify genetic loci that may increase the risk of idiopathic ONFH. In total, 217 patients with idiopathic ONFH and 217 control samples, without ONFH, were genotyped using Axiom ${ }^{\mathrm{TM}}$ chips. Following quality control, 509,886 single-nucleotide polymorphisms (SNPs) were included in the association analysis to identify genetic variants that may influence susceptibility to idiopathic ONFH. The lowest P-value identified by the current study was for an association with rs220324 $\left(\mathrm{P}=3.57 \times 10^{-7}\right)$, an SNP that is located near to the uromodulin-like 1 gene region on chromosome 21q22.3, although none of the SNPs reached the traditional genome-wide significance level of $5 \times 10^{-8}$. However, the DnaJ heat shock protein family (Hsp40) member C6 (DNAJC6) locus, a region between 65.37 and $65.67 \mathrm{Mb}$ located on chromosome $1 \mathrm{p} 31.3$, harbored a cluster of SNPs that were associated with idiopathic ONFH at a significance level of $\mathrm{P}<1 \times 10^{-5}$. Four
\end{abstract}

Correspondence to: Professor Shin-Yoon Kim, Department of Orthopedic Surgery, Graduate School of Medicine, Kyungpook National University, 101 Dongin-dong 2 Ga, Daegu 41944, Republic of Korea

E-mail: syukim@knu.ac.kr

Dr Tae-Ho Kim, Biomedical Research Institute, Kyungpook National University Hospital, 135 Dongdeok-ro, Daegu 41940, Republic of Korea

E-mail: kimth0929@ynu.ac.kr

Key words: osteonecrosis of the femoral head, genome-wide association, polymorphism, idiopathic variants, rs10493374, rs12032616, rs17127529 and rs6679032, with marginal associations were located in and around the DNAJC6 locus and were in strong linkage disequilibrium with each other. In conclusion, the current study did not identify any SNPs that were associated with idiopathic ONFH at a genome-wide significance level, however, the results suggest that future studies should investigate the effects of SNPs in the DNAJC6 gene on the idiopathic ONFH risk.

\section{Introduction}

Osteonecrosis is the death of bone tissue following microvascular injury, which results in bone resorption and, potentially, structural collapse. The disease typically affects the epiphyseal bone on the convex side of a joint, which may be due to a lack of collateral circulation and most commonly affects the femoral head, although it may also affect the humeral head, femoral condyles, proximal tibia, vertebra, and small bones of the hand and foot (1). Osteonecrosis of the femoral head $(\mathrm{ONFH})$ is a complex multifactorial disease that is associated with genetic predisposition and exposure to certain environmental factors. Various etiological factors, including the use of corticosteroids, alcohol abuse, sickle cell anemia, radiation and Gaucher disease, are known to be implicated in the development of secondary osteonecrosis (2). It is possible for certain patients, those who have received high doses of corticosteroids or consumed excessive amounts of alcohol for a long period of time, to develop ONFH, however, there are also rare cases of ONFH that occur following short-duration corticosteroid treatment, thus indicating that differences exist in the susceptibility to risk factors and the genetic predisposition to ONFH between individuals $(3,4)$. Idiopathic ONFH is diagnosed when individuals are not known to have been exposed to any of the known risk factors. The incidence or prevalence of idiopathic ONFH reflects ethnic differences (5). Idiopathic ONFH in identical twins and the clustering of cases in families indicate that genetic factors may be involved in development $(6,7)$. Several studies have investigated genetic traits that may predispose an individual to ONFH development, including those involved in clotting disorders (8-10). However, many of the results of genetic studies investigating the association between genes involved in the clotting 
process and the risk of ONFH in Caucasians have not been replicated in other ethnic groups. For example, factor V Leiden and prothrombin G20210A mutations have not been identified in the Korean population $(11,12)$. This suggests that geographic and ethnic differences exist in the prevalence of disease-associated genes and/or polymorphisms. In addition, Liu et al reported that all patients with familial ONFH in the study carried collagen type II $\alpha 1$ chain (COL2A1) mutations, whereas no mutations were identified in the COL2A1 coding region in patients with sporadic ONFH (13). The majority of ONFH cases are sporadic, therefore, it is essential to screen candidate genes from a different perspective. Various genetic methods have been developed to identify disease-associated loci or causal variations associated with specific diseases. Genome-wide association studies (GWAS) have been facilitated by the availability of chip-based microarray techniques, which allow assays of more than a million single-nucleotide polymorphisms (SNPs) in order to identify disease-associated loci and potential causal variants associated with diseases. GWAS has been used to map a huge number of susceptibility genes for numerous complex diseases, including type 1 and type 2 diabetes, inflammatory bowel disease, prostate cancer and breast cancer (14-16). Since GWAS was first reported in 2005, 2,041 studies have been added to the Catalog of Published GWAS (http://www.genome.gov/26525384), however, to the best of our knowledge, GWAS of patients with idiopathic ONFH has not previously been performed. GWAS was performed in the present study to identify genetic variants that influence susceptibility to and outcomes of idiopathic $\mathrm{ONFH}$ in the Korean population.

\section{Materials and methods}

Study subjects. The study was initially performed at the GWAS discovery stage to identify SNPs potentially involved in the development of idiopathic ONFH in the Korean population. The ONFH patients were recruited consecutively from Kyungpook National University Hospital (Daegu, Korea) between 2002 and 2012, and written informed consent was obtained from all study participants prior to enrolment. This study was approved by the Institutional Review Board of the Kyungpook National University Hospital. All patients with ONFH were diagnosed by an orthopedist according to the diagnostic criteria of the Association Research Circulation Osseous classification system based on magnetic resonance imaging and plain radiographs (17). Based on etiological factors, patients were assigned to one of the following groups: Idiopathic, steroid-induced and alcohol-induced osteonecrosis. Only the patients in the idiopathic group were included in this study. Control subjects were defined by a lack of hip pain and the absence of any lesions with sclerotic margins or subchondral collapse consistent with ONFH in anteroposterior and frog-leg lateral pelvic radiographs. The GWAS included 217 idiopathic ONFH (152 males and 65 females aged $49.5 \pm 14.2$ years) and 217 control ( 57 males and 160 females aged $54.7 \pm 12.8$ years) samples.

Genome-wide SNP genotyping. Genomic DNA was extracted from whole human blood using the FlexiGene DNA kit (Qiagen, Inc., Valencia, CA, USA) according to the manufacturer's protocol. For each sample, genotyping was performed with the Axiom ${ }^{\circledR} 2.0$ genome-wide ASI 1 Array kit (Affymetrix, Inc., Santa Clara, CA, USA) according to the standard protocols recommended by the manufacturer. Briefly, for each array, $200 \mathrm{ng}$ genomic DNA was amplified and fragmented randomly into fragments of 25-125 base pairs (bp). The initial amplification had a reaction volume of $40 \mu \mathrm{l}$, which contained $20 \mu \mathrm{l}$ genomic DNA at a concentration of $10 \mathrm{ng} / \mu \mathrm{l}$ and $20 \mu \mathrm{l}$ Denaturation Master Mix (reagent from Module 1(P/N901711) of the Axiom 2.0 reagent kit). The initial amplification was performed for $10 \mathrm{~min}$ at room temperature. Following the initial amplification, the incubated products were amplified with $130 \mu$ Axiom $^{\circledR} 2.0$ Neutral Solution, $225 \mu 1$ Axiom $^{\circledR} 2.0$ Amp Solution, and $5 \mu 1$ Axiom $^{\circledR}$ 2.0 Amp Enzyme. Amplification reactions were performed for $23 \pm 1 \mathrm{~h}$ at $37^{\circ} \mathrm{C}$. Amplification products were then used to amplify fragments of 200-1,100 bp. A fragmentation step reduced the amplified products into segments of $\sim 25-50 \mathrm{bp}$, which were then end-labeled using reagents from Module 2-1(P/N901528) and Module 2-2(P/N901529) of the Axiom 2.0 Reagent kit. Following hybridization, the bound target was washed under stringent conditions to remove non-specific background and minimize any background noise caused by random ligation events (reagents from Module 4-1(P/N901278) and Module 4-2(P/N901276) of the Axiom 2.0 Reagent kit). Each polymorphic nucleotide was queried via a multi-color ligation event performed on the array surface. Following ligation, the arrays were stained and imaged using a GeneTitan ${ }^{\circledR}$ Multi-Channel Instrument (Affymetrix, Inc.). Images were analyzed using Genotyping Console ${ }^{\mathrm{TM}}$ software 4.14 (Affymetrix, Inc.).

Genotype quality control. Raw data in the form of CEL files were imported into Affymetrix Power Tools (version 1.16.1; Affymetrix, Inc.) and genotype calling was performed using Axiom GT1 algorithm (Affymetrix, Inc.). Samples with dish quality control (QC) value of $>0.82$ and call rate $>0.95$ were considered to have passed the quality control assessment. Downstream analysis was performed using PLINK (http://pngu.mgh.harvard.edu/ purcell/plink/) version no. 1.07 (18). Markers were considered not to meet the quality control criteria and discarded if they were demonstrated to have any of the following: Call rate $<95 \%$, minor allele frequency of the control $<1 \%$, and deviations from the Hardy-Weinberg equilibrium with a P-value $<10^{-7}$. It was confirmed that the call rate per sample was $>97 \%$.

Statistical analysis. Statistical analyses were performed using PLINK (version no. 1.07) (18) and SAS (version no. 9.1.3; SAS Institute, Cary, NC, USA). To test for allelic and genotype associations between SNPs and ONFH, 2x2 and $2 \times 3$ contingency tables were constructed, which were compared using Cochran-Armitage trend, Chi-squared, and Jonckheere-Terpstra tests. The strength of association was estimated by the odds ratio and the $95 \%$ confidence interval, which were calculated using Cornfield methods. Three genetic models of inheritance (recessive, dominant, and codominant) for SNPs were assessed. Genotypes were given codes of 0,1 and 2; 0,1 and 1; and 0,0 and 1 in the codominant, dominant, and recessive models, respectively. The quantile-quantile (Q-Q) plot demonstrates close agreement 


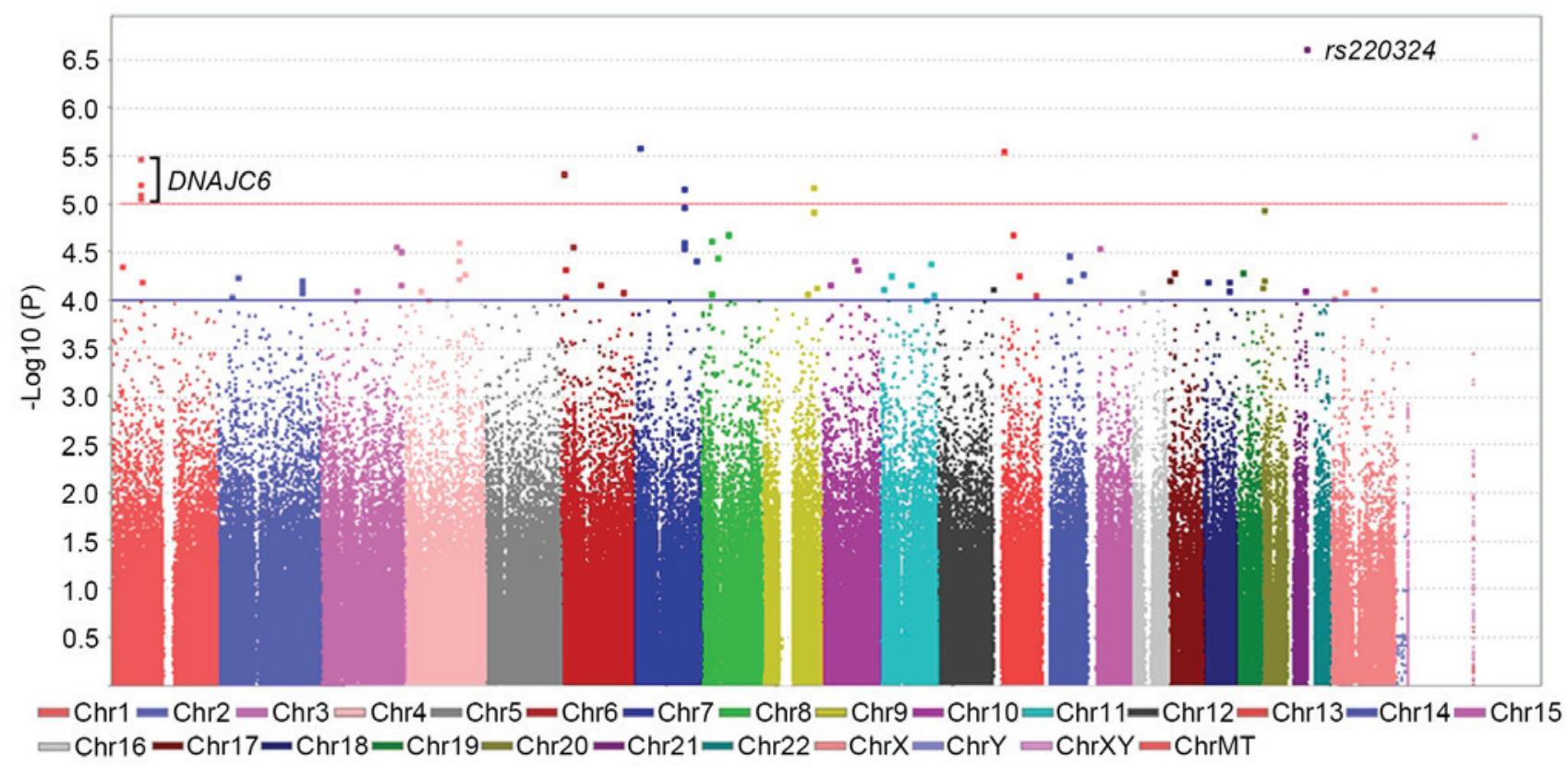

Figure 1. Genome-wide SNP association signals for idiopathic ONFH. Manhattan plot, logarithm of P-values vs. Chr coordinates, of SNP associations in idiopathic ONFH. The red and blue lines denote P-values of $1 \times 10^{-5}$ and $1 \times 10^{-4}$, respectively. SNP, single-nucleotide polymorphism; ONFH, osteonecrosis of the femoral head; Chr, chromosome.

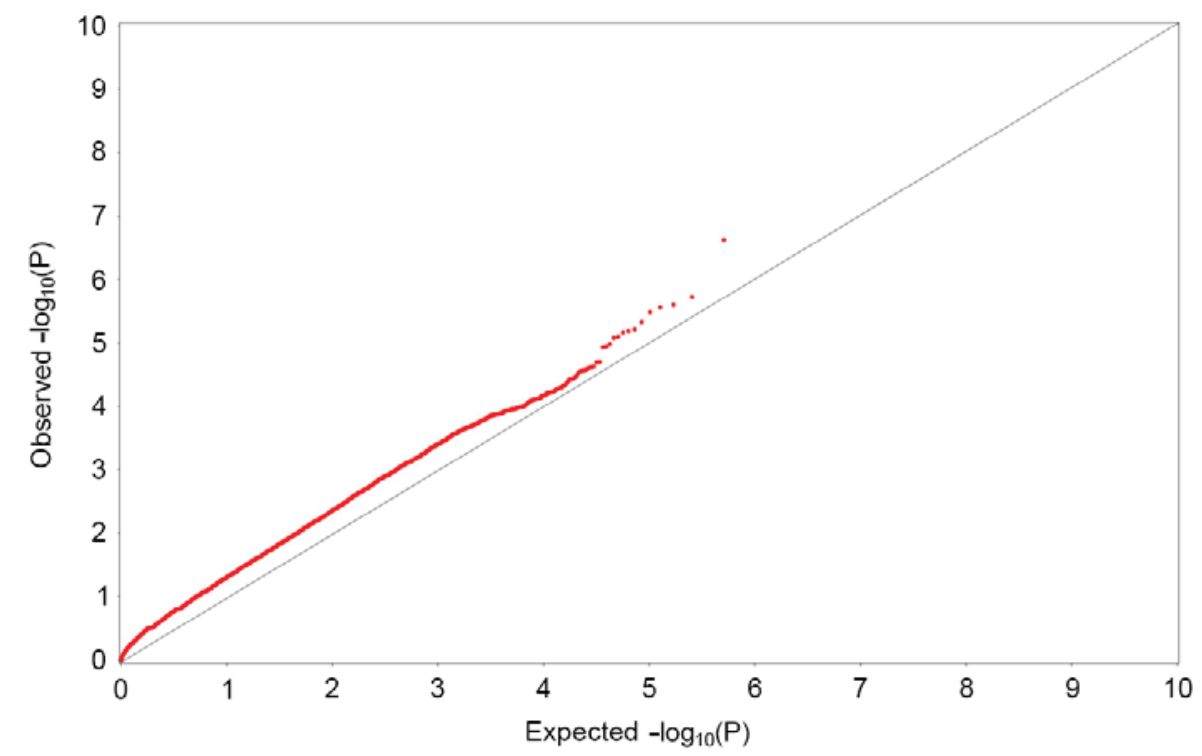

Figure 2. Quantile-quantile plot of genome-wide association study results for idiopathic osteonecrosis of the femoral head. The horizontal axis indicates the expected $-\log 10$ (P-values). The vertical axis indicates the observed - $\log 10$ (P-values). The gray line represents $\mathrm{y}=\mathrm{x}$.

between two distributions if the population stratification and cryptic relatedness are controlled adequately. Undetected population stratification or cryptic relatedness results in deviation from the null across the entire distribution; whereas, large-effect susceptibility loci generate deviations at the highly significant end of the range (19). Regional association plots were created using LocusZoom (version no. 1.3; https://statgen.sph.umich.edu/locuszoom/genform. php?type=yourdata; University of Michigan, Ann Arbor, MI, USA). Haploview (version no. 4.2; http://www.broad.mit. edu/mpg/haploview; Broad Institute, Cambridge, MA, USA) was used to determine linkage disequilibrium (LD) in the genomic region using an accelerated expectation-maximization algorithm (20).

\section{Results}

Genotype data for 217 idiopathic ONFH cases (152 male, 65 female) and 217 control (57 male, 160 female) subjects was analyzed. Based on stringent quality control criteria, 509,886 SNPs were selected for association analysis for idiopathic ONFH. A Manhattan plot of the association analyses for all SNPs was produced using the chromosomal positions (x-axis) and negative logarithm of P-values (y-axis) for each 


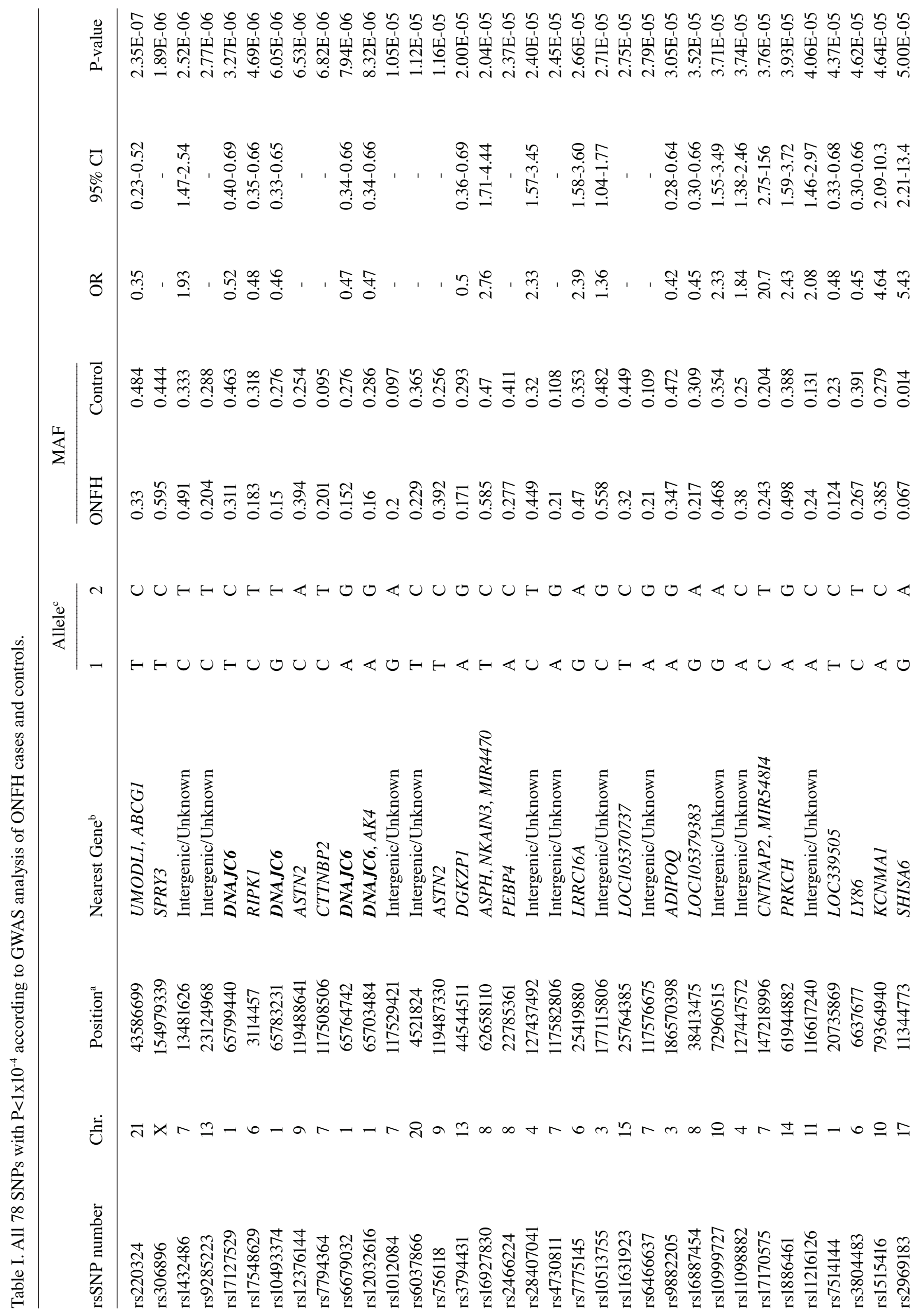




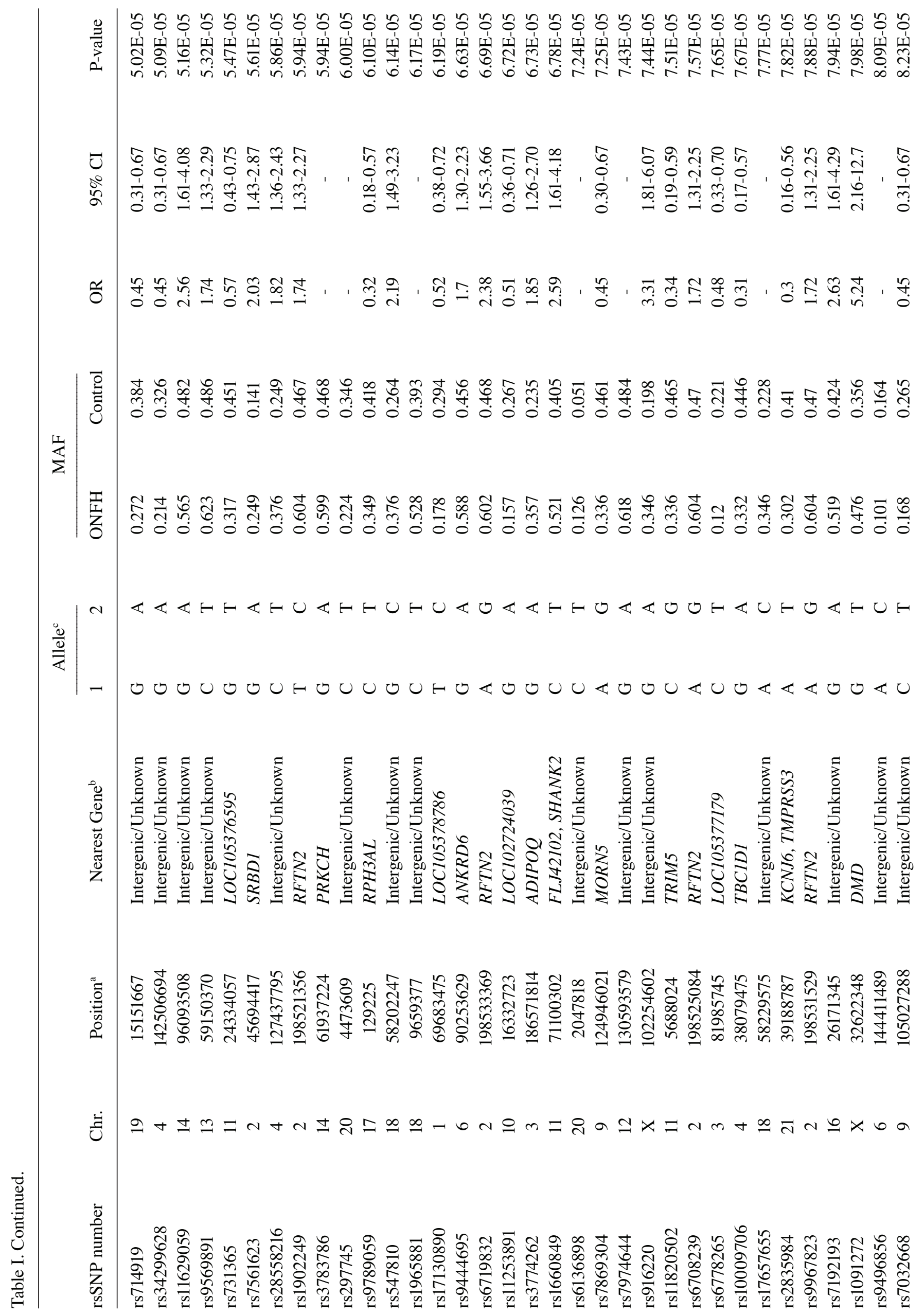


SNP (Fig. 1). In the Q-Q plot (Fig. 2), minimal deviation from the expected P-value distribution was observed, and only at the upper tail of the distribution, suggesting that population stratification was adequately controlled. All of the association signals with $\mathrm{P}<1 \times 10^{-4}$ (78 SNPs) are shown in Table I. Although none of the SNPs reached the accepted genome-wide significance level of $5 \times 10^{-8}, 11$ SNPs on chromosomes 1,6 , 7, 9, 13 and 21 had the strongest associations with increased susceptibility to idiopathic ONFH at a significance level of $\mathrm{P}<10^{-5}$ (Fig. 1 and Table I). The most significant association was detected at rs220324 $\left(\mathrm{P}=3.57 \times 10^{-7}\right)$, which is located in an intergenic region between the uromodulin-like 1 (UMODLI) gene and the ATP-binding cassette, sub-family G, member 1 $(A B C G 1)$ gene region on chromosome 21q22.3. Given that certain disease-associated genes may be enriched among the top ranked genes in GWAS, the present study then filtered the SNPs to identify chromosomal regions that harbored clusters of associated SNPs at a less stringent criterion $\left(\mathrm{P}<1 \times 10^{-5}\right)$. It was observed that DnaJ heat shock protein family (Hsp40) member C6 (DNAJC6), a region between 65.37 and $65.67 \mathrm{Mb}$ in the chromosome $1 \mathrm{p} 31.3$ region, harbored a cluster of SNPs, which were associated with idiopathic ONFH at a significance level of $\mathrm{P}<1 \times 10^{-5}$. Four significant SNPs, rs10493374, rs12032616, rs17127529 and rs6679032 in the DNAJC6 gene region were revealed to be in strong LD with each other (Table II), and all had associations with idiopathic ONFH at a significance level of $\mathrm{P}<1 \times 10^{-5}$. The regional association plot and $\mathrm{LD}$ plot for these loci are presented in Fig. 3.

\section{Discussion}

ONFH usually affects young adults and, without treatment, progresses to the collapse of femoral head leading to osteoarthritis and destruction of the hip joint (21). ONFH is one of the most common diseases of the hip joint in Korea, where its incidence is relatively high compared with other countries $(5,22)$. Approximately $50-60 \%$ of total primary hip replacements are thought to be the result of ONFH in Korea (5). In Korea, non-traumatic ONFH is associated with idiopathic factors in $36 \%$, alcoholism in 35\%, steroids in $14 \%$ and other factors in $15 \%$ of cases (23). In a previous study, Kang et al (22) reported the prevalence of ONFH in Korea using medical claims data obtained from the Korean National Health Insurance Service (Wonju, Korea). The estimated average number of annual cases was 14,103 , thus, indicating an average prevalence of 28.91 per 100,000 over a 5-year period (2002-2006). It was demonstrated that $32.4 \%$ had a history of alcohol abuse and $14.6 \%$ of cases were associated with steroid usage. In recent years, the clinical significance of ONFH for hip diseases has received attention, however, the details of its pathogenesis and epidemiology are not well understood; although it is generally assumed that venous thrombosis resulting in blood flow obstruction to the femoral head, mediated by thrombophilia and/or hypofibrinolysis, is important in the development of ONFH $(24,25)$. Several studies have investigated the genetic traits that may predispose subjects to ONFH development. However, contrary to expectations, the results of various studies have demonstrated that differences are observed between reports with different study designs and/or ethnic groups involved in the analyses. 
Table II. Linkage disequilibrium relationships (ID'l and $\mathrm{r}^{2}$ ) between the four SNPs that had strong associations with the DNAJC6 gene.

\begin{tabular}{lcccc}
\hline & \multicolumn{3}{c}{ SNP ID'| } \\
\cline { 2 - 5 } SNP r $^{2}$ & $r s 12032616$ & $r s 6679032$ & $r s 10493374$ & $r s 17127529$ \\
\hline$r s 12032616$ & & 0.860 & 0.860 & 0.749 \\
$r s 6679032$ & 0.687 & 1 & 1 & 1 \\
$r$ s10493374 & 0.685 & 0.428 & 0.426 & 1 \\
$r$ s17127529 & 0.256 & & \\
\hline
\end{tabular}

SNP, single nucleotide polymorphism; DNAJC6, DnaJ heat shock protein family (Hsp40) member C6.

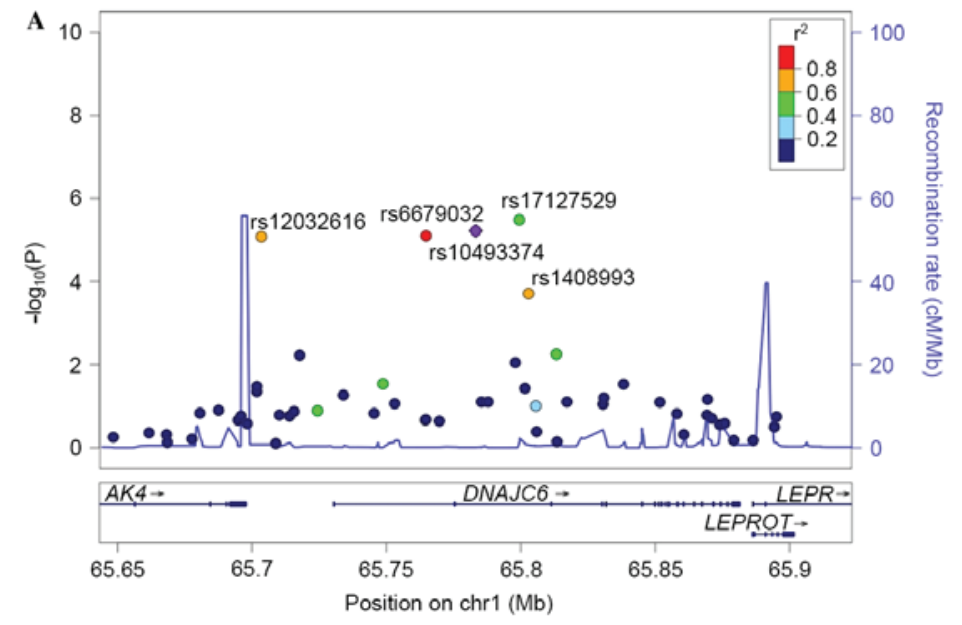

B

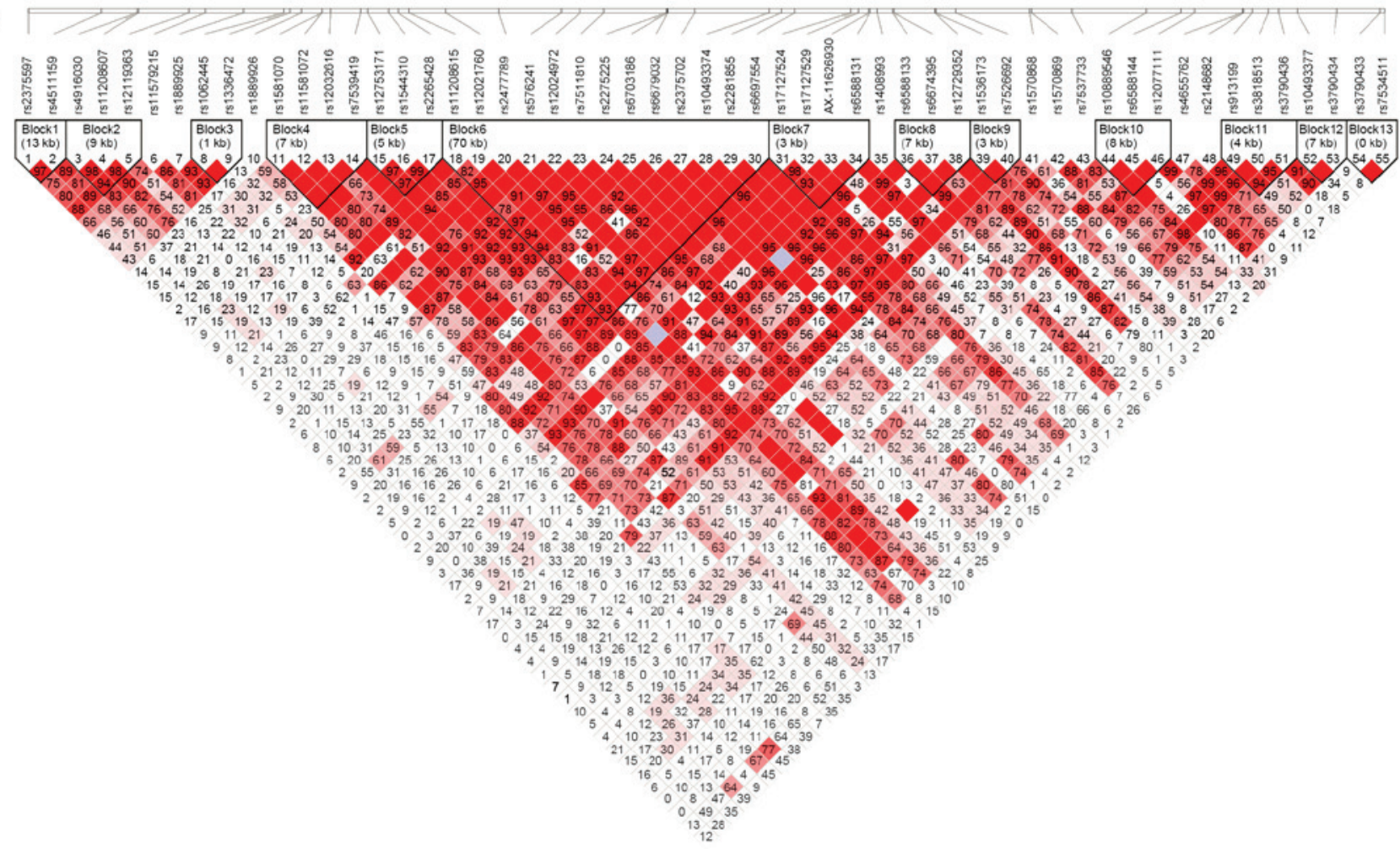

Figure 3. Regional plot of SNPs in the DNAJC6 locus and the LD relationship among these SNPs. (A) Data are shown for the DNAJC6 locus around the four associated SNPs. The rs17127529 SNP in the regional plot was the SNP with the most significant association with idiopathic ONFH in the DNAJC6 gene. The blue curve highlights the recombination rates based on 1000 Genomes Asian data. (B) The strength of the LD relationship (D') between the most strongly associated SNP and the other SNPs is represented by the red color intensity based on genome-wide association study data from the present study. SNP, single-nucleotide polymorphism; LD, linkage disequilibrium; Chr, chromosome. 
The present study conducted a GWAS in the Korean population to identify new susceptibility loci associated with idiopathic ONFH. To the best of our knowledge, it is the first high-density GWA scan of ONFH in a Korean population, and the lowest P-value, and most significant association, this study identified was obtained for the association of idiopathic ONFH with rs220324 $\left(\mathrm{P}=3.57 \times 10^{-7}\right)$, which is located in an intergenic region between the UMODL1 gene and $A B C G 1$ gene region on chromosome 21q22.3. Notably, the cholesterol transporter ABCG1 is involved in cholesterol homeostasis, where it promotes cholesterol efflux from cells and regulates intracellular cholesterol homeostasis (26). However, none of the SNPs identified by the present study satisfied the recognized GWA criteria $\left(\mathrm{P}<1 \times 10^{-8}\right)$. Given that certain disease-associated genes may be enriched among the top ranked genes in GWAS, the present study searched for chromosomal regions containing two or more significant SNPs within $100 \mathrm{~kb}$ of the significant marker SNPs using a less stringent P-value, and the LD structure of the region was analyzed using GWAS data. Subsequently, a chromosomal block containing genes that clustered with SNPs thought to be associated with ONFH $\left(\mathrm{P}<1 \times 10^{-5}\right)$ was identified. The four variants, rs10493374, rs12032616, rs17127529 and rs6679032, with modest associations were located in and around the DNAJC6 locus. A previous GWAS of the anticoagulant pathway also detected significant associations between certain SNPs in the DNAJC6 gene and free protein S plasma levels (27). The protein $\mathrm{C}$ anticoagulant pathway regulates blood coagulation by preventing the formation of thrombi. This pathway has two main plasma components, protein $\mathrm{C}$ and protein $\mathrm{S}$. Deficiencies in antithrombin, protein $\mathrm{C}$ and protein $\mathrm{S}$, or an impaired anticoagulant pathway, increase the incidence of thromboembolic disorders (27). Notably, a number of researchers have suggested that intravascular coagulation and microcirculatory thrombotic occlusion may provide a common pathway to non-traumatic osteonecrosis. It is assumed that venous thrombosis with blood flow obstruction to the femoral head, mediated by thrombophilia and/or hypofibrinolysis, leads to increased intraosseous pressure, reduced arterial flow and hypoxia, which appear to be important in the development of ONFH $(24,25)$. The exact function of DNAJC6 remains unknown, however, the protein encoded by DNAJC6 resembles a tyrosine-protein phosphatase auxilin, which is an enzyme that promotes the uncoating of clathrin-coated vesicles, thus, it potentially has a role in endocytosis. Endocytosis itself, which is followed by partial proteolysis, is involved in coagulation via molecular modification of factor V and factor VIII $(27,28)$. Thus, a similar mechanism involving DNAJC6 and free protein $\mathrm{S}$ plasma levels may be associated with ONFH, although validation of this hypothesis requires further investigation. When a less stringent $\mathrm{P}$-value $\left(\mathrm{P}<1 \times 10^{-4}\right)$ was applied to select candidate regions for further investigation, in phase 2 of the present study, several chromosomal regions that clustered with SNPs thought to be associated with idiopathic ONFH were discovered (data not shown). The present study performed one of the first GWAS of idiopathic ONFH in Koreans and aimed to identify genetic variants that influence susceptibility to idiopathic ONFH. The results indicated that several SNPs in the DNAJC6 gene are potentially associated with idiopathic ONFH. Further investigations, including replicate studies, are required to confirm whether DNAJC6 is an ONFH susceptibility gene, but the preliminary findings of the present study provide novel insight into the genetic factors associated with the risk of ONFH.

\section{Acknowledgements}

This research was supported by Biomedical Research Institute grant, Kyungpook National University Hospital (grant no. 15-04; 2015), and partially supported by a by a grant of the Korea Health Technology R\&D Project through the Korea Health Industry Development Institute, funded by the Ministry of Health \& Welfare, Republic of Korea (grant no. HI15C0001).

\section{References}

1. Nishimura T, Matsumoto $\mathrm{T}$, Nishino $\mathrm{M}$ and Tomita $\mathrm{K}$ : Histopathologic study of veins in steroid treated rabbits. Clin Orthop Relat Res 334: 37-42, 1997.

2. Assouline-Dayan Y, Chang C, Greenspan A, Shoenfeld Y and Gershwin ME: Pathogenesis and natural history of osteonecrosis. Semin Arthritis Rheum 32: 94-124, 2002.

3. Orlić D, Jovanović S, Anticević D and Zecević J: Frequency of idiopathic aseptic necrosis in medically treated alcoholics. Int Orthop 14: 383-386, 1990.

4. Jones LC and Hungerford DS: The pathogenesis of osteonecrosis. Instr Course Lect 56: 179-196, 2007.

5. Kim SY and Rubash HE: Avascular necrosis of the femoral head: the Korean experience. In: The adult hip. Vol 2.2nd edition. Lippincott Williams \& Wilkins, Philadelphia, PA, pp1078-1086, 2006.

6. Glueck CJ, Glueck HI, Welch M, Freiberg R, Tracy T, Hamer T and Stroop D: Familial idiopathic osteonecrosis mediated by familial hypofibrinolysis with high levels of plasminogen activator inhibitor. Thromb Haemost 71: 195-198, 1994.

7. Nobillot R, Le Parc JM, Benoit J and Paolaggi JB: Idiopathic osteonecrosis of the hip in twins. Ann Rheum Dis 53: 702, 1994.

8. Ferrari P, Schroeder V, Anderson S, Kocovic L, Vogt B, Schiesser D, Marti HP, Ganz R, Frey FJ and Kohler HP: Association of plasminogen activator inhibitor-1 genotype with avascular osteonecrosis in steroid-treated renal allograft recipients. Transplantation 74: $1147-1152,2002$.

9. Zalavras CG, Vartholomatos G, Dokou E and Malizos KN: Factor V Leiden and prothrombin gene mutations in femoral head osteonecrosis. Thromb Haemost 87: 1079-1080, 2002.

10. Zalavras CG, Malizos KN, Dokou E and Vartholomatos G: The $677 \mathrm{C}-\mathrm{T}$ mutation of the methylene-tetrahydrofolate reductase gene in the pathogenesis of osteonecrosis of the femoral head. Haematologica 87: 111-112, 2002.

11. Kim SY, Suh JS, Park EK, Jung WB, Kim JW, Koo KH and Kim CY: Factor V Leiden gene mutation in femoral head osteonecrosis. J Korean Ortho Res Soc 6: 259-264, 2003.

12. Chang JD, Hur M, Lee SS, Yoo JH and Lee KM: Genetic background of nontraumatic osteonecrosis of the femoral head in the Korean population. Clin Orthop Relat Res 466: 1041-1046, 2008.

13. Liu YF, Chen WM, Lin YF, Yang RC, Lin MW, Li LH, Chang YH, Jou YS, Lin PY, Su JS, et al: Type II collagen gene variants and inherited osteonecrosis of the femoral head. N Engl J Med 352: 2294-2301, 2005.

14. Gordon H, Trier Moller F, Andersen V and Harbord M: Heritability in inflammatory bowel disease: From the first twin study to genome-wide association studies. Inflamm Bowel Dis 21: 1428-1434, 2015.

15. Veron A, Blein S and Cox DG: Genome-wide association studies and the clinic: A focus on breast cancer. Biomark Med 8: 287-296, 2014.

16. Grarup N, Sandholt CH, Hansen T and Pedersen O: Genetic susceptibility to type 2 diabetes and obesity: from genome-wide association studies to rare variants and beyond. Diabetologia 57: 1528-1541, 2014.

17. Gardeniers JWM: The Arco Perspective for Reaching One Uniform Staging System of Osteonecrosis. In: Bone Circulation and Vascularization in Normal and Pathological Conditions. Scoutens A, Arlet J, Gardeniers JWM and Hughes SPF (eds). Vol 247. Plenum Press, New York, NY, pp375-380, 1993.

18. Purcell S, Neale B, Todd-Brown K, Thomas L, Ferreira MA, Bender D, Maller J, Sklar P, de Bakker PI, Daly MJ and Sham PC: PLINK: A tool set for whole-genome association and population-based linkage analyses. Am J Hum Genet 81: 559-575, 2007. 
19. Clayton DG, Walker NM, Smyth DJ, Pask R, Cooper JD, Maier LM, Smink LJ, Lam AC, Ovington NR, Stevens HE, et al: Population structure, differential bias and genomic control in a large-scale, case-control association study. Nat Genet 37: 1243-1246, 2005.

20. Barrett JC, Fry B, Maller J and Daly MJ: Haploview: Analysis and visualization of LD and haplotype maps. Bioinformatics 21 : $263-265,2005$.

21. Glimcher MJ and Kenzora JE: The biology of osteonecrosis of the human femoral head and its clinical implications. III. Discussion of the etiology and genesis of the pathological sequelae; commments on treatment. Clin Orthop Relat Res 140: 273-312, 1979.

22. Kang JS, Park S, Song JH, Jung YY, Cho MR and Rhyu KH: Prevalence of osteonecrosis of the femoral head: A nationwide epidemiologic analysis in Korea. J Arthroplasty 24: 1178-1183, 2009.

23. Koo KH, Jeong ST and Jones JP Jr: Borderline necrosis of the femoral head. Clin Orthop Relat Res 358: 158-165, 1999.
24. Jones LC, Mont MA, Le TB, Petri M, Hungerford DS, Wang P and Glueck CJ: Procoagulants and osteonecrosis. J Rheumatol 30: 783-791, 2003.

25. Kerachian MA, Harvey EJ, Cournoyer D, Chow TY and Séguin C: Avascular necrosis of the femoral head: Vascular hypotheses. Endothelium 13: 237-244, 2006.

26. Sag D, Cekic C, Wu R, Linden J and Hedrick CC: The cholesterol transporter ABCG1 links cholesterol homeostasis and tumour immunity. Nat Commun 6: 6354, 2015.

27. Athanasiadis G, Buil A, Souto JC, Borrell M, López S, Martinez-Perez A, Lathrop M, Fontcuberta J, Almasy L and Soria JM: A genome-wide association study of the Protein C anticoagulant pathway. PLoS One 6: e29168, 2011.

28. Camire RM and Bos MH: The molecular basis of factor V and VIII procofactor activation. J Thromb Haemost 7: 1951-1961, 2009. 